\title{
Characterization of risk of depression and suicide in a sample of elderly people in Beja, Portugal - an exploratory study
}

\author{
Sónia Farinha-Silva ${ }^{1}$, Paulo Barbosa ${ }^{1}$, Maria Teresa Valadas ${ }^{1}$, Tomás Matos-Pires $^{2}$, Marta Moreira ${ }^{3}$, Ana Matos-Pires ${ }^{1}$ \\ 1 Unidade Local de Saúde do Baixo Alentejo, Psychiatry, Beja, Portugal \\ ${ }^{2}$ Faculdade de Ciências Sociais e Humanas - Universidade Nova de Lisboa, Geography and Regional Planning, \\ Lisboa, Portugal \\ ${ }^{3}$ Faculdade de Psicologia da Universidade de Lisboa, Psychology, Lisboa, Portugal
}

Suicide has a strong impact on public health, not only because it is generally associated with treatable pathology like major depressive disorder, but also because it has a dramatic impact on survivors. Suicide is a growing problem in our country, and Beja, a district in the interior south of Portugal, is among the regions with the highest suicide rates. The issues of rurality, isolation and old age are known suicide risk factors that are important in Beja, where $25 \%$ of the population is over 65 years old.

We decided to conduct an exploratory study aiming to characterize depression and suicide among elderly people in this country.

Inclusion criteria, subjects:

- Over 65 years old

- Residing in the county of Beja

- Who accepted to participate in the study

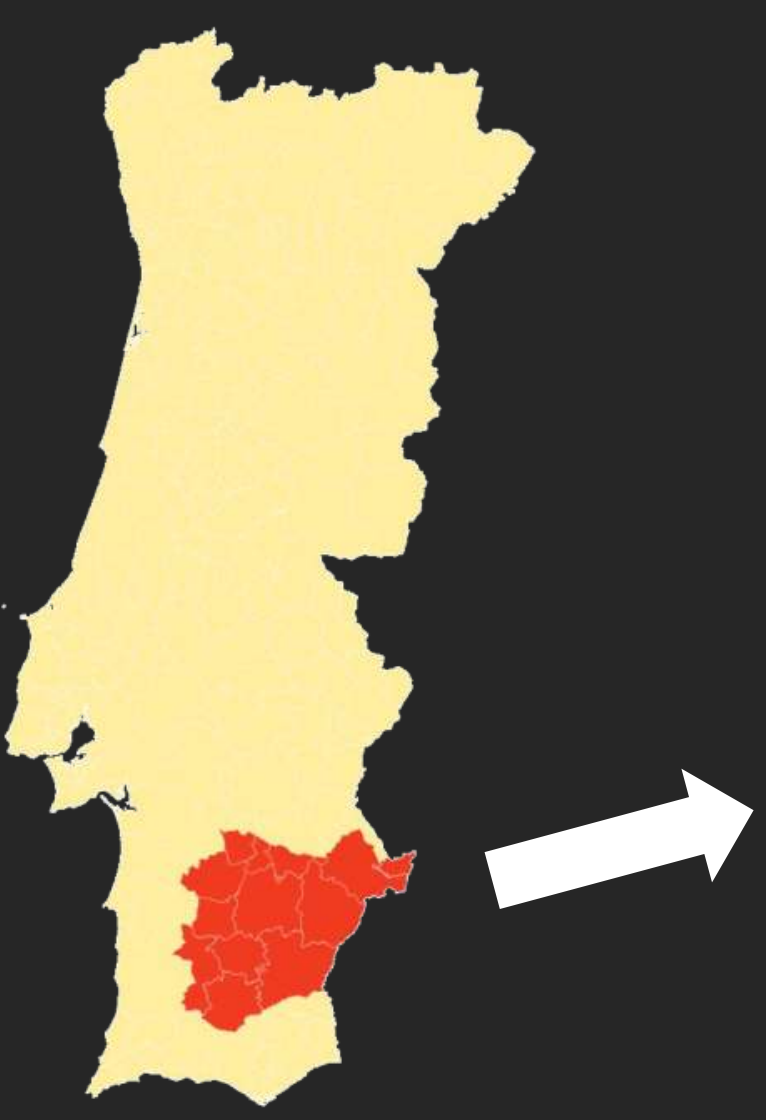

Beja district

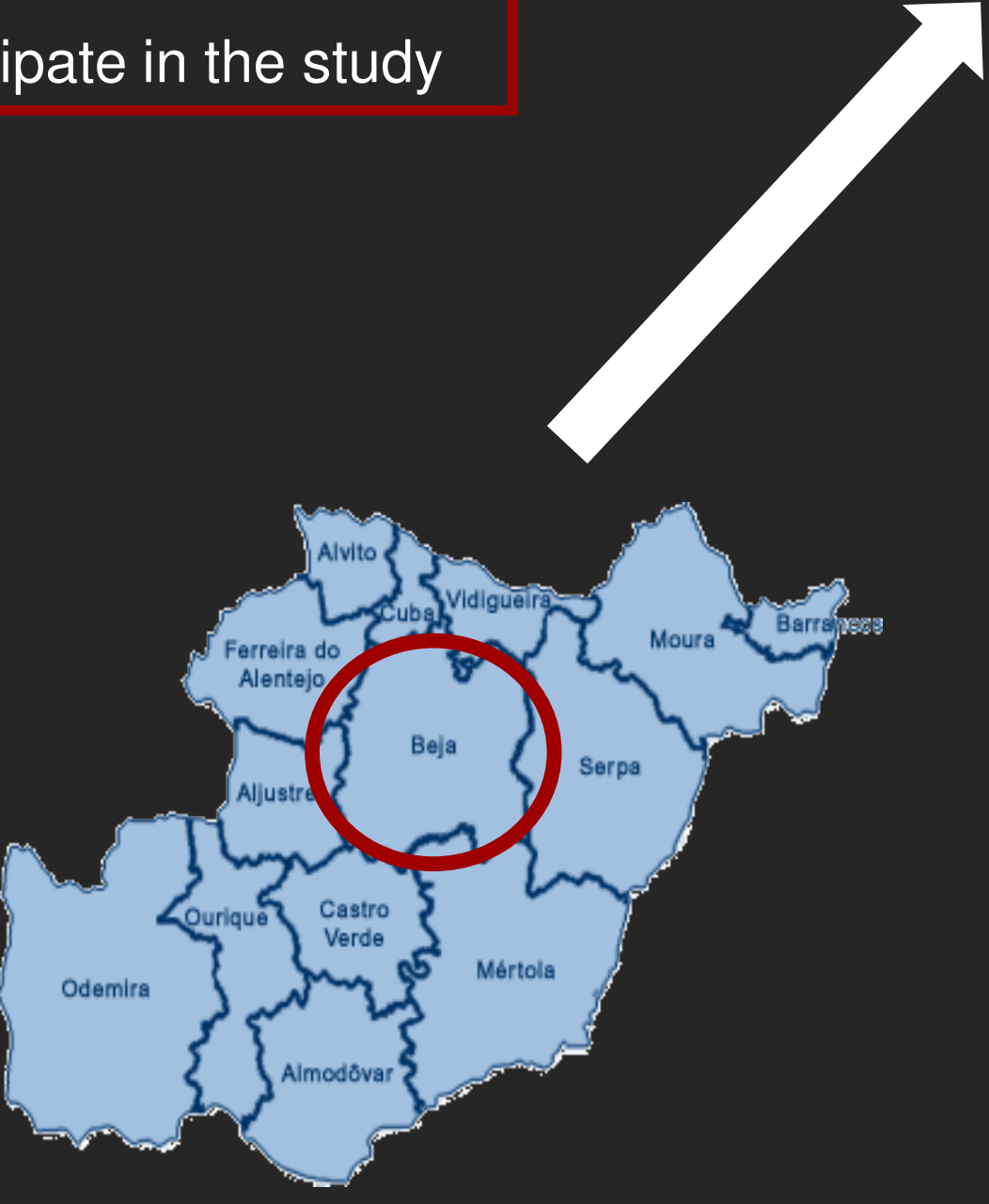

Beja county
Elderly people (>65 years old) in the county

of Beja $(\mathbf{n}=7562)$
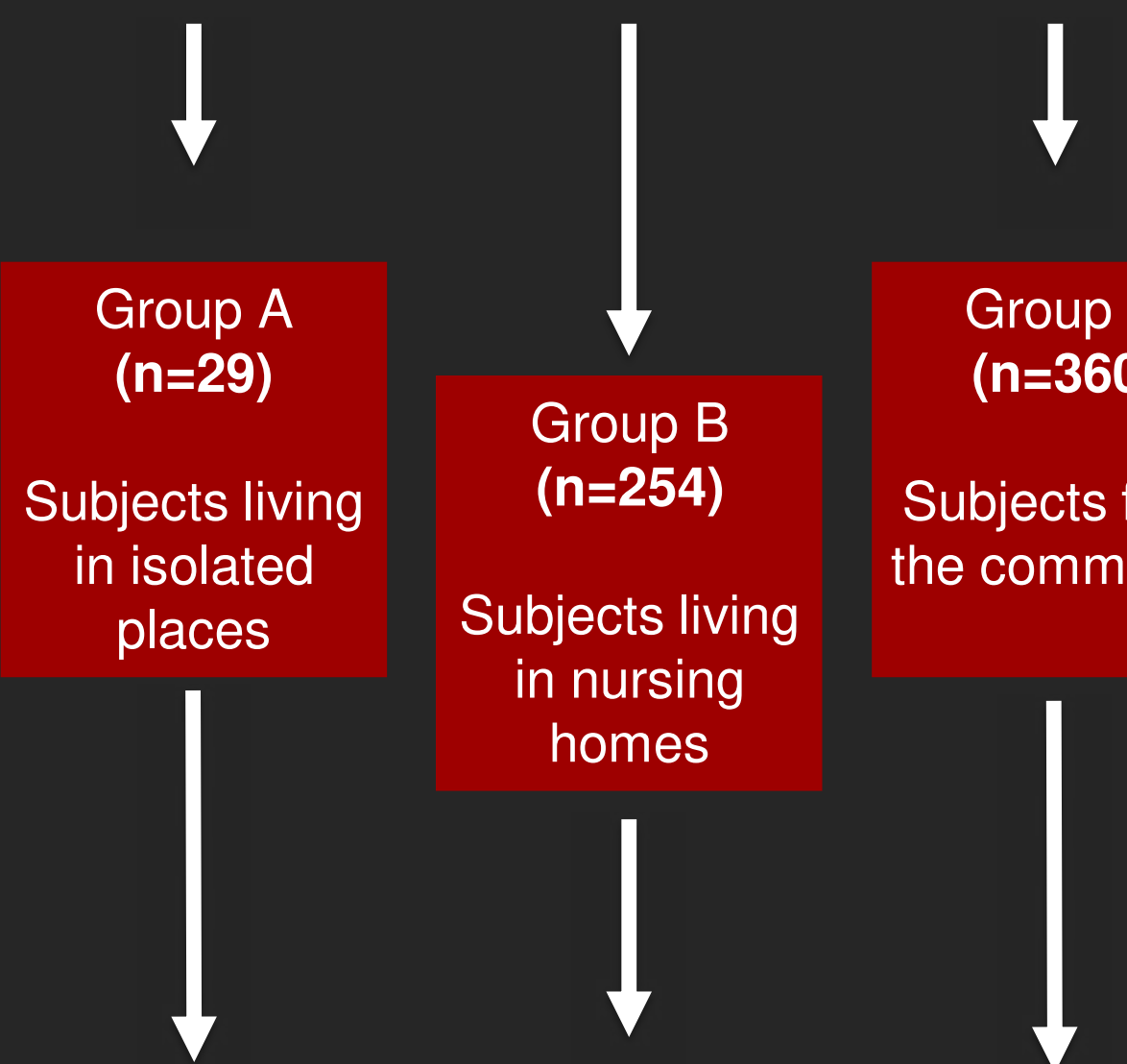

Group C $(n=360)$

Subjects from the community

Sociodemographic questionnaire Geriatric Depression Scale

Columbia Suicide Severity Rating Scale WHO Quality of Life-BREF

\section{Results}

We tried to evaluate a representative sample from each group, but we ended up collecting the following data regarding subjects that accepted to participate in the study: Group A - total number of subjects living in isolated places; Group B data from all of the subjects living in nursing homes excluding two nursing homes which did not cooperate; Group C - a representative sample of subjects evaluated in primary health care units and old age psychiatry outpatient setting.

\section{Conclusions}

By conducting the first exploratory study of this kind in our country, we hope to better understand the specific characteristics and risk factors of our elderly population, in order to develop specific programs for intervention in depression and suicide prevention. 